\title{
Valoración del hormigón ante ataque de sulfatos
}

\author{
Assessment of concrete against sulfate attack
}

\author{
Avaliação de concreto contra ataque de sulfato
}

\author{
Franz Javier Catur Muraña \\ franzjaviercaturmurana@gmail.com \\ ORCID 0000-0002-8631-8815
}

Universidad Autónoma Tomás Frías, Potosí-Bolivia

Artículo recibido enero 2021 | Arbitrado en febrero 2021 | Publicado en mayo 2021

\section{RESUMEN}

Dada la importante participación actual del hormigón como material de construcción, se estudia bastante su comportamiento, influencia, variación y durabilidad. El objetivo de este artículo fue evaluar el efecto de diferentes tipos de materiales de impermeabilización en el rendimiento del hormigón expuesto a ataque por sulfatos; mediante un diseño experimental que midió la evolución de su degradación estando expuesto a diferentes variaciones físicas y químicas, durante 180 días. Como resultado se determinó que el desprendimiento del hormigón frente a acciones químicas en suelos agresivos y condiciones ambientes puede ser mitigado mediante el mejoramiento de su densidad y una adecuada protección por materiales impermeabilizantes. Se concluyó que el curado del hormigón es esencial para combatir estos ataques y la aplicación de materiales impermeabilizante puede mejorar significativamente la durabilidad del hormigón.

Palabras clave: Hormigón; Física; Química; Experimento; Humedad; Resistencia

ABSTRACT

RESUMO

Given the important current participation of concrete as a construction material, its behavior, influence, variation and durability are studied quite a lot. The objective of this article was to evaluate the effect of different types of waterproofing materials on the performance of work exposed to an attack by sulfates; by means of an experimental design that measured the evolution of its degradation in a state exposed to different physical and chemical variations, during 180 days. As a result, it was determined that the detachment of concrete against chemical actions in aggressive soils and ambient conditions can be mitigated by improving its density and adequate protection by waterproofing materials. It was concluded that the curing of concrete is essential to combat attacks and the application of waterproofing materials can significantly improve the durability of concrete.

Key words: Concrete; Physical; Chemistry; Experiment; Humidity; Endurance
Dada a importante participação atual do concreto como material de construção, seu comportamento, influência, variação e durabilidade são muito estudados. 0 objetivo deste artigo foi avaliar o efeito de diferentes tipos de impermeabilizantes no desempenho de concretos expostos ao ataque de sulfatos; por meio de um desenho experimental que mediu a evolução de sua degradação sendo exposto a diferentes variações físicas e químicas, durante 180 dias. Como resultado, determinou-se que o desprendimento do concreto contra ações químicas em solos agressivos e condições ambientais pode ser mitigado pela melhoria de sua densidade e proteção adequada por materiais impermeabilizantes. Concluiu-se que a cura do concreto é fundamental para o combate a esses ataques e a aplicação de impermeabilizantes pode melhorar significativamente a durabilidade do concreto.

Palavras-chave: Concreto; Fisica; Química; Experimentar; Umidade; Resistência 


\section{INTRODUCCIÓN}

Dada la importante participación del hormigón como material de construcción, se estudia bastante su comportamiento, influencia, variación y durabilidad; respeto a la durabilidad. Los ataques de sulfatos tanto en superficie expuesta como en fundaciones tienen implicaciones considerables.

$\mathrm{Si}$ bien el ataque puede ser categorizado como químico, surge de una serie de reacciones entre los iones de sulfato y productos de hidratación o componentes del cemento, que son objeto de varias investigaciones (American Concrete Institute, 2001). Por otra parte, el ataque físico denominado como una meteorización de sal, genera presiones contra la pared de los poros que inducen principalmente al escalonado en la superficie del hormigón (Hayner y Bassuoni, 2012), las principales acciones de corrosión física son el sulfato de sodio, carbonato de sodio, y cloruro de sodio; sin embargo, el mecanismo no se conoce completamente (American Concrete Institute, 2001). A pesar de que estos procedimientos son aceptados, existe un debate sobre el tipo de ataque que gobierna y las condiciones; el mecanismo puede tener un gran número de variaciones.

Según las orientaciones actuales de las normas como ACI (American Concrete Institute), la durabilidad es una propiedad fundamental para el diseño de estructuras; en una estructura de hormigón es su capacidad para soportar durante su vida útil condiciones físicas y químicas a las que está expuesta (Ministerio de Fomento, 2011).

Los ataques sulfatos son un conjunto complejo de procesos químicos y físicos entre los iones de sulfato y los componentes del hormigón, principalmente la pasta de cemento, provenientes de una fuente externa o interna, como la exposición del hormigón a sulfatos y humedad (Neville, 2004), es todavía un tema que promueve una gran cantidad de discusión. La discusión puede causar la división con diferentes formas de pensar y resolver problemas. Araujo (2013) se señalan tres compuestos que desempeñan un papel importante en las reacciones: a) Sulfato de calcio, conocido como yeso (Gypsum) CaSO $4 * 2 \mathrm{H}_{2} \mathrm{O}$; b); b) Sulfoaluminato de calcio, conocido como la etringita (Ettringite 3CaO. Al2O3CaSO432H2O); c) El calcio silicio sulfocarbonate, conocido como taumasita CaSiO2. CaCO3CaSO $415 \mathrm{H} 2 \mathrm{O}$.

Aunque de acuerdo con la ACI 201 (American Concrete Institute, 2001) existen dos mecanismos que pueden ser considerados como ataques sulfatos: la formación de yeso (gypsum) y la formación de etringita. Se considera que el producto de ambas reacciones puede provocar daños en el hormigón por incrementar el volumen total de sólidos (American Concrete Institute, 2001). Estas reacciones (yeso y etringita) son las más conocidas, siendo objetivo de varias investigaciones (Collepardi, 2002) (Jara Del Rosario, 2016); sin embargo, una simple división requerida debe hacerse en relación con el ataque por sulfatos, la diferencia ente ataque de sulfatos interna (ASI) y ataque de sulfatos externa (ESA). Una diferencia se puede explicar en la generación de etringita primaria que se produce inmediatamente (en horas) en una mezcla fresca de plástico que no produce ninguna expansión perjudicial y se asocia con la regulación del tiempo de la pasta de cemento portland ajuste; en cambio, la etringita secundaria es 
la que ocurre después del endurecimiento de la muestra de hormigón (Collepardi, 2002).

Tal como se ha expuesto previamente, el estudio de la durabilidad del hormigón es complejo y amerita el desarrollo de diversas investigaciones. En este sentido, el objetivo de este artículo fue evaluar el efecto de diferentes tipos de materiales de impermeabilización en el rendimiento del hormigón expuestos a ataque por sulfatos. La principal utilidad que respalda este estudio experimental es poder contar con métricas adecuadas a variables del entorno: temperatura, humedad y materiales principalmente.

\section{MATERIALES Y MÉTODO}

Se trató de una investigación de tipo cuantitativo y alcance experimental que tuvo por objetivo evaluar el efecto de diferentes tipos de materiales de impermeabilización en el rendimiento del hormigón expuestos a ataque por sulfatos. La hipótesis planteada fue: la protección por materiales de impermeabilización en la superficie del hormigón mejora su durabilidad bajo ciertas condiciones de exposición a sulfatos. El experimento se desarrolló siguiendo la norma $\mathrm{ABC}$ (Administradora Boliviana de Carreteras, 2007).

Entre los instrumentos usados en el experimento, fue necesaria una cámara ambiental que modele el comportamiento térmico registrado por logger, un congelador como cámara ambiental que registró las temperaturas bajas, y una cámara diseñada específicamente para registrar temperaturas altas. Asimismo, se contó con termostato (MH1210N220V), humidificador Mist Maker y Datalogger HT20.
Siguiendo la norma ABC, el experimento consistió en probar cuatro tipos de materiales en tratamiento de superficie con una aplicación crítica de dos pasadas de sellado, los materiales se caracterizan por ser repelentes a agua: alquitrán modificado; poliuret plastificante para pisos de alto tránsito; recuplast para techo (rojo), de gran elasticidad, alta resistencia mecánica y resistente a los factores climáticos; recuplast ladrillo, que evita la penetración irreversible de manchas y escrituras, evita la formación de hongos, no saponifica.

Se realizaron cuatro experimentos para mejorar la viscosidad del alquitrán, se mezcló alquitrán con; aceite de motor, diésel, gasolina y querosene. Como resultado se encontró la manera más fácil de impermeabilizar la superficie del hormigón, mejorando bastante la mezcla de alquitrán (50\%) con gasolina (50\%) a temperaturas ambiente de $25^{\circ} \mathrm{C}$, así el alquitrán modificado resultó ser adecuado para el revestimiento con brocha en la superficie del hormigón.

Se evaluaron materiales impermeabilizantes: alquitrán modificado, poliuret, recuplat techo, recuplat ladrillo. Se estudió el rendimiento mecánico en un ensayo de corrosión acelerada por solución de sulfato de sodio al $6 \%$, en ciclos de calor/seco y frio/húmedo. Se introdujo sulfato de corrosión externa para simular el entorno. Se sometieron probetas a laboratorio, con los diferentes materiales impermeabilizantes y relaciones de a/c de 0.45 y 0.55 . Además, se realizaron réplicas de las probetas para evaluar su resistencia con agua potable y aguas con iones de sulfato. 


\section{RESULTADOS}

En este apartado se describe el trabajo realizado, partiendo de la descripción del experimento y concluyendo con la evaluación de los resultados en cuatro dimensiones: resistencia; ataque de los sulfatos; efectos ante materiales de impermeabilización; apariencia visual.

\section{Estudio experimental}

Para la evaluación se consideraron los siguientes criterios; mayoración cinco veces más los iones de sulfato encontrados en el suelo; un $6 \%$ de $\mathrm{Na}_{2} \mathrm{SO}_{4}$; se trabajaron sesenta cilindros de hormigón en total, con un diámetro de $10 \mathrm{~cm}$ y una altura de $20 \mathrm{~cm}$ de acuerdo con Montoya (2010). En primera instancia, se separaron grupos en los que las variables en el diseño de mezcla fueron de relación a/c 0.45 y 0.55; la resistencia a la compresión dosificada de los cilindros a los 28 días de $210 \mathrm{~kg} / \mathrm{cm} 2$; un mesclador mecánico y fundido en cilindros de tubería PVC de 4in; el procedimiento de vaciado de las probetas contempló dosificación según Montoya (2010).

Asimismo, se utilizaron agregados de la zona: grueso, seleccionado con $19 \mathrm{~mm}$ de tamaño máximo de partículas, densidad real del árido seco y absorción 2.8 y 0.469\% respectivamente; fino, arena graduada de río con una densidad real del árido seco, absorción y módulo de finura de 2.46, $1.275 \%$ y 3.41 respectivamente. Se usó cemento PI-30 con contenido C3A del 9\%.

Para simular una sobresaturación, condición necesaria del mecanismo de daño a la cristalización del agente corrosivo, se usaron dos cámaras ambientales, las temperaturas altas fueron simuladas con bombillas incandescentes y un reflector controlado por un termostato; las temperaturas bajar mediante un equipo refrigerante controlado manualmente.

Las temperatura-humedades a calibrar fueron $[6-8]^{\circ} \mathrm{C} /[90-95] \%$; se tomaron registros de temperatura dentro del equipo refrigerante en grados de enfriamiento 1, 2, 3 , observando que en grado de enfriamiento 2 se lograba variaciones de temperaturas adecuadas. Con las humedades relativas entre intervalos 50 $60 \%$ fue necesario aumentar la humedad produciendo oscilaciones eléctricas a nivel de frecuencias ultrasónicas. Sin embargo, no se logró uniformidad.

Para controlar la uniformidad dentro de la cámara refrigerante se utilizó un ventilador de computadora ubicado por debajo del equipo humidificador a la altura de las probetas expuestas; a partir de esto se obtuvo calibración uniforme y constante dentro de la cámara. El humidificador estuvo activo durante 25 minutos, luego se contó con temperaturas calibradas sin necesidad de éste.

Las temperatura altas y humedades bajas a calibrar fueron $[36-38]^{\circ} \mathrm{C} /[20-$ 25]\%; se empleó un reflector con potencia de $500 \mathrm{~W}$; la calibración se realizó a una altura de $25 \mathrm{~cm}$ inmediatamente por encima del nivel de las probetas sobre la que se definió una grilla acorde al reflejo, esta altura se escogió precisamente para abarcar temperaturas hasta los estrechos del lado más corto. Esto se controló por el termostato desde temperaturas ambiente $\mathrm{LP}=20^{\circ} \mathrm{C}$ hasta los $\mathrm{HA}=(36-38)^{\circ} \mathrm{C}$, entre los radios de giro de temperaturas $\mathrm{CP}=16 \mathrm{y}$ $18^{\circ} \mathrm{C}$. El tiempo y la variación de temperaturas se presentan en la Tabla 1. 
Tabla 1. Calibración de temperatura dentro de la cámara.

\begin{tabular}{|c|c|c|}
\hline \multirow[b]{2}{*}{ Nro. } & t a $36^{\circ} \mathrm{C}$ & t a $38^{\circ} \mathrm{C}$ \\
\hline & \multicolumn{2}{|c|}{$\mathbf{t}[\mathrm{min}]$} \\
\hline 1 & 1.5 & 2.06 \\
\hline 2 & 1.55 & 2.14 \\
\hline 3 & 1.53 & 2.08 \\
\hline 4 & 1.56 & 2.12 \\
\hline 5 & 1.54 & 2.1 \\
\hline 6 & 1.54 & 2.09 \\
\hline 7 & 1.57 & 2.13 \\
\hline 8 & 1.58 & 2.16 \\
\hline 9 & 1.53 & 2.08 \\
\hline 10 & 1.49 & 2.05 \\
\hline prom $=$ & 1.54 & 2.101 \\
\hline
\end{tabular}

La Tabla 1 muestra la calibración en un tiempo promedio $\mathrm{t}=2.1$ minutos, abarca el área uniforme entre las variaciones de temperatura $(36-38)^{\circ} \mathrm{C}$, los registros a $36^{\circ} \mathrm{C}$ en lados lejanos y de $38^{\circ} \mathrm{C}$ grados en la parte central, según Figura 1.

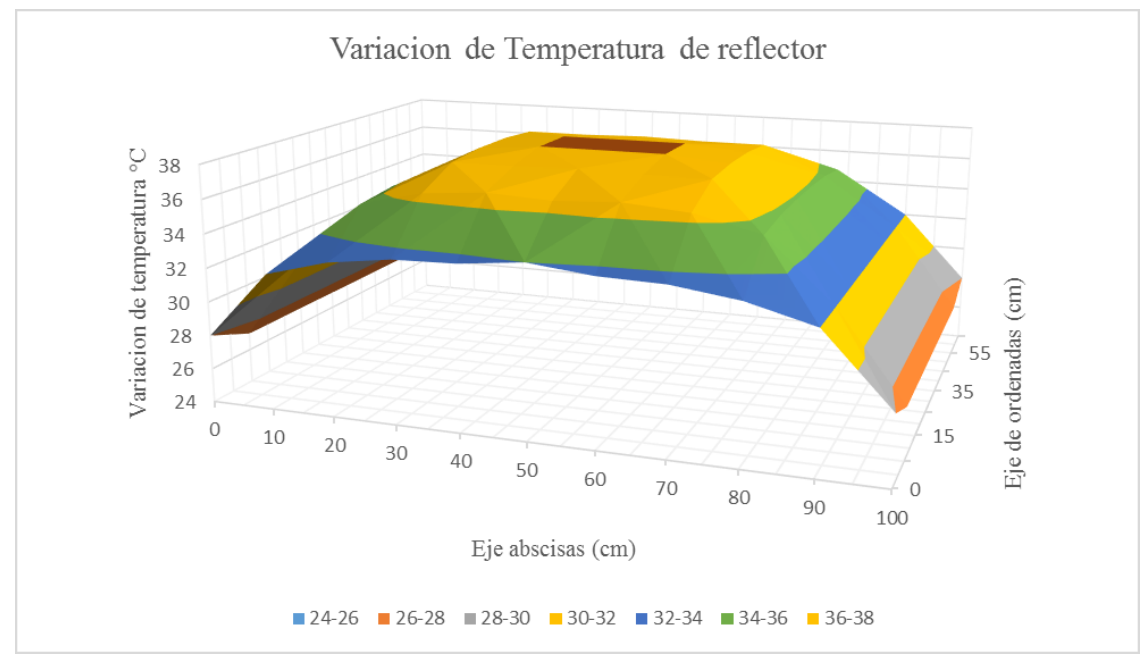

Figura 1. Vista isométrica de variación temperatura en $\mathrm{t}=2.1$ (primera prueba)

Como se ve en la Figura 1, no se consiguió las temperaturas deseadas en los extremos. Esto se solucionó con la incorporación de bombillas incandescentes de $60 \mathrm{~W}$. La calibración tomó como parámetro el tprom $=2.1 \mathrm{~min}$, la variable que se controló en este caso es la altura, llegando a la temperatura en $\mathrm{h}=5 \mathrm{~cm}$ por encima de la superficie calibrada, el registro del área que abarca se muestra en la Figura 2. 


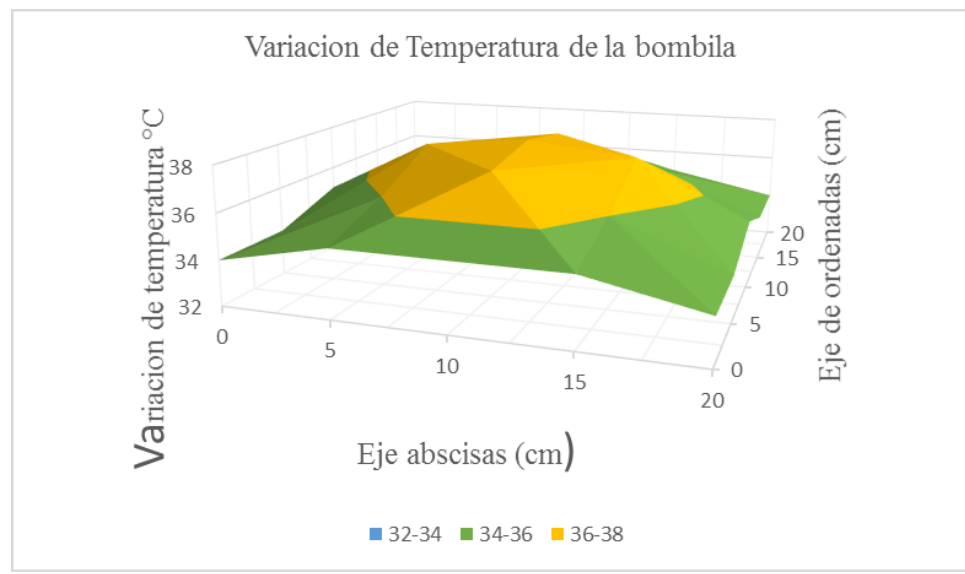

Figura 2. Vista isométrica de variación temperatura en $\mathrm{t}=2.1 \mathrm{~min}$ (segunda prueba)

Para uniformizar las temperaturas se utilizaron un ventilador, inicialmente a nivel del agua. Se realizaron varias mediciones a diferentes alturas. Finalmente, se obtuvo humedades mínimas dentro de la cámara con apertura de tapa y ubicando el ventilador a altura de probeta; Se obtuvo las calibraciones de temperaturas mostradas en la Figura 2.

Esquema de ensayos de ataque de sulfatos acelerado, los cilindros de hormigón estuvieron recubiertos siete días de curado, tal como recomienda ACI 201 (Montoya, 2010), usando agregados duros y resistencias n menores de 41Mpa. Después de un curado de las probetas de siete días como mínimo se dejaron en un ambiente de $20^{\circ} \mathrm{C}$ y humedades menores a $50 \%$ durante $48 \mathrm{~h}$. Posteriormente, las muestras se sometieron a una concentración de 6 de sulfato hasta un tercio de su altura.

Asimismo, las muestras se sometieron a condiciones de temperaturas $y$ humedades cíclicas basadas en los diagramas de fase de sulfato de sodio para provocar una cristalización repetitiva y la generación de la sobresaturación. La exposición continuó durante 3 meses, con ciclos de 12 horas consecutivas de fase de calor/seca por 4 horas $\left(36-38^{\circ} \mathrm{C}\right.$ y $20-25 \%$ de humedad relativa), siguiendo por 8 horas de frío/húmedo $\left(6-8^{\circ} \mathrm{C}\right.$ y $90-95 \%$ de humedad relativa).

Dieciocho probetas de hormigón fueron construidas para la verificación de la resistencia a compresión con variables de a/c, agua potable y agua recogida del lugar. Veintinueve probetas de hormigón fueron evaluadas en laboratorio, veinticuatro de ellas con una dosificación óptima admitida según normas ACI Committee 201 (American Concrete Institute, 2001) e Instituto Boliviano de Normalización y Calidad (1987). con relación a/c de 0.45, se adoptó una mezcla seca competente a agresiones químicas teóricamente y cinco de ellas con relación a/c de 0.55

Se evaluaron: propiedades físicomecánicas; cambios mecánicos a 0, 60, 180 ciclos; resistencia mecánica de las probetas replicadas y las probetas expuesta a 180 ciclos de cambios ambientales de calor/frío, tanto con las relaciones de a/c 0.55 y 0.45 , a 60 ciclos, donde las probetas expuestas a laboratorio estarían a 39 días después del vaciado; resistencia por un método aproximado no destructivo.

Al final del curado de 7 días como mínimo según las recomendaciones establecidas anteriormente o cero ciclos, las 
réplicas con las relaciones agua/cemento se ensayaron para la resistencia a compresión de acuerdo con (UNE-EN-12390-3, 2003).

Para evaluar la resistencia mecánica de las probetas de hormigón a los 60 ciclos se utilizó la técnica de ensayo no destructivo, prueba de esclerómetro en la variabilidad de la resistencia, acuerdo con las recomendaciones de (UNE-EN-12504-2, 2002). Las probetas fabricadas con las relaciones de agua/cemento de 0.45 y 0.55 inicialmente mostraron en su esquema de poros, el tamaño de los poros de las probetas con relación agua/cemento 0.55 fue mucho mayor a la de las probetas con relación de agua/cemento de 0.45 .

Durante la exposición a ataque de sulfatos en laboratorio, las muestras se controlaron en intervalos regulares. Después de un periodo de exposición de calor/frío la eflorescencia de sal y los desechos se retiraron cuidadosamente de la superficie de la muestra utilizando un cepillo de nylon; se dejaron secar en condiciones de laboratorio dentro de la cámara para la modelación en caliente a temperatura de $20^{\circ} \mathrm{C}$ y las humedades relativas menores de 50\%. Para la evaluación visual se aplicó una escala numérica de 0 (sin escala) a 5 (escala grave), de manera similar a las encontrada en la norma de ASTM C672 (Corral, 2009). Se clasificó también el proceso de degradación para el control de calificación visual.

Las probetas de hormigón fueron transmitidas a condición de exposición después de medir su masa inicial usando una balanza con una precisión $0.1 \mathrm{~g}$ [0.05oz], antes de exponer a laboratorio, los controles respectivos de la masa se armaron a 0, 30, 60, 120, 180 ciclos. Las probetas de hormigón fueron secadas a $30^{\circ} \mathrm{C}$ y humedades relativas menores a $50 \%$. El cambio de masa se calculó de acuerdo con la ecuación:

Pérdida de masa $(t)=\frac{M_{i}+M_{t}}{M_{i}} * 100$

Para identificar los eflorescentes se utilizó un análisis de difracción de rayos $\mathrm{x}$ (XRD); las muestras fueron tomadas de la superficie de las probetas deterioradas.

\section{Evaluación de efectos de resistencia}

Los resultados de la capacidad de carga de las probetas vaciadas con agua potable y aguas que contienen iones de sulfato recogido del lugar fueron evaluados para ver el comportamiento mecánico y hacer una comparativa de la influencia de su utilización en la resistencia a compresión y curado. La evaluación de los componentes químicos en laboratorio tuvo como resultado $2.88 \%$ de $\mathrm{Na}_{2} \mathrm{SO}_{4}$; en el proceso de vaciado mostró las formaciones de eflorescencia inmediata. Después de los 27 días de curado en la intemperie se mostró la formación de macro poros.

La formación de etringita primaria tuvo notable efecto en las 24 horas después del vaciado: formación de yeso en la intemperie de las probetas y la formación de poros capilares o macro poros; además, la reducción de resistencia con respecto a las probetas utilizadas con agua potable redujo al $35.69 \%$.

\section{Efecto de ataque de los sulfatos en las propiedades mecánicas del hormigón}

Los efectos de resistencia se resumen en: variación en la capacidad de soporte de carga de las probetas bajo la acción de los 
ciclos de calor/frío sometidos a soluciones de sulfato de sodio; la resistencia a compresión de las probetas replicadas se controló a 7 días de curado, es decir, 0 ciclos y también se controla la resistencia de las probetas degradadas al final de la exposición; las resistencia de compresión, también tiende a controlarse a los 28 días, tras someterse a 7 días de curado a los 28 días posiblemente no llegue la resistencia dosificada por la importancia del curado, además de haber pasado 60 ciclos bajo las acciones destructivas en laboratorio, para esto se controló una evaluación no destructiva, tal como se muestra en la Figura 3.

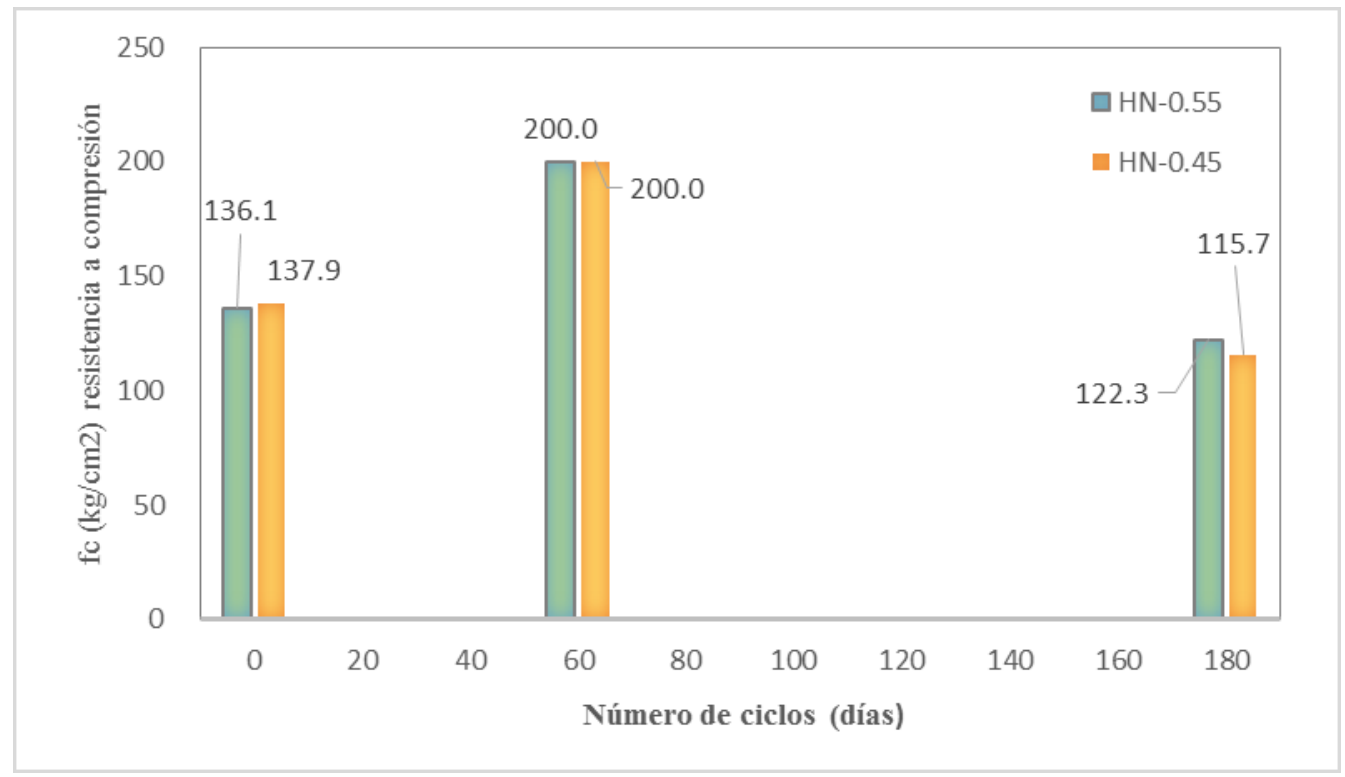

Figura 3. Variación de la resistencia tras los 180 ciclos- ataque de sulfatos.

Según se observa en la Figura 3, el comportamiento de las probetas de hormigón en la evaluación en su estado inicial y final muestra el decaimiento de la resistencia, tras los ciclos de exposición es notablemente significativa.

\section{Efecto ante materiales de impermeabilización en el comportamiento mecánico sulfato atacado}

El comportamiento mecánico de las probetas de hormigón fue evaluado. En la Figura 4 se presentan sus resistencias en estado final tras haber pasado los 180 ciclos en laboratorio de calor/frío. 


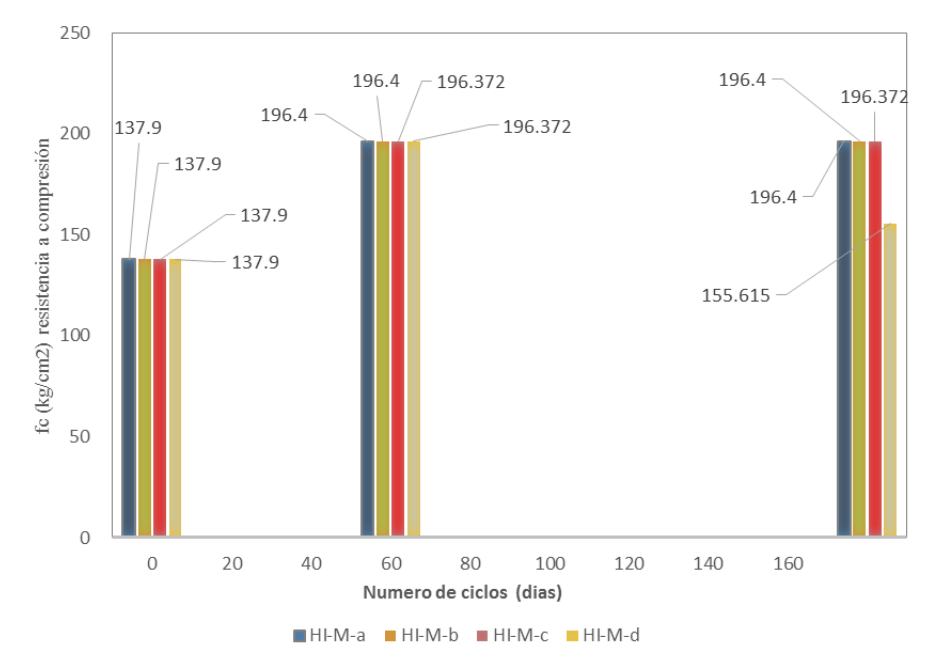

Figura 4. Variación de la resistencia tras los 180 ciclos - materiales de impermeabilización

El comportamiento demostrado en la Figura 4 representa los resultados de las probetas con materiales impermeabilizantes que no se dañan y llegan a una resistencia de $196.37 \mathrm{~kg} / \mathrm{cm} 2$, esto es efecto de curar solo 7 días; en cambio, el material recuplat ladrillo tuvo un decaimiento considerable por el fracaso del material como revestimiento.

\section{Evaluación visual}

De acuerdo con los diagramas de fase de sulfato de sodio, las probetas de hormigón en laboratorio expuesta a cambio de frío a caliente dos veces en $24 \mathrm{~h}$ experimentaron deposición de sal en la superficie; la eflorescencia comenzó a crecer sobre todo en la parte de secado, siguiendo a un escalonado progresivo y la descamación. El deterioro del hormigón y el escalamiento comenzó por encima del nivel de solución y luego se propagó hacia la parte superior. A partir de los 120 ciclos las muestras se redujeron considerablemente como consecuencia del escalonamiento progresivo en la superficie del hormigón, la secuencia de deterioro de las probetas en el tiempo se muestra en la figura 5 para las relaciones a/c bajos y para la a/c alto en la figura 6 . 

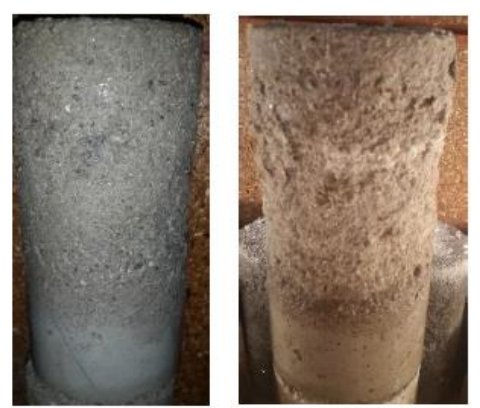

0 dias

30 días

60 dias

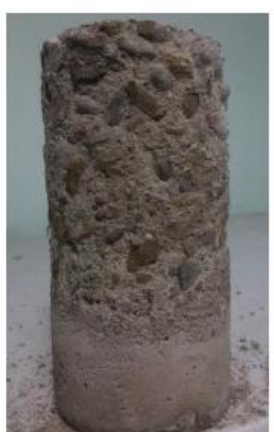

120 dias

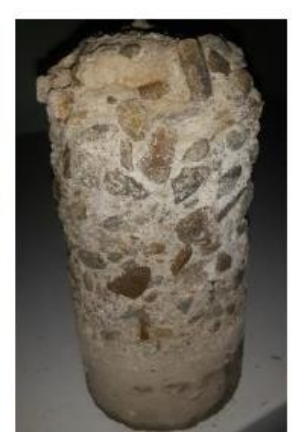

180 días

Figura 5. Degradación progresiva de la muestra con relación de a/c 0.45

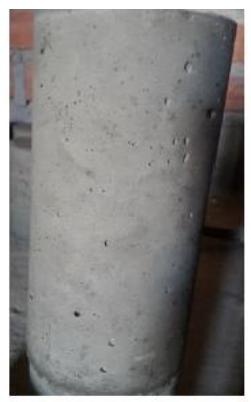

0 dias

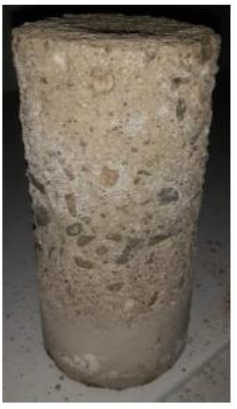

120 dias

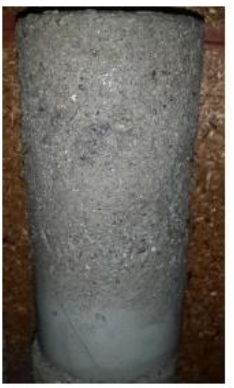

30 dias

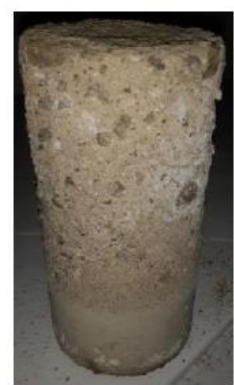

60 días

Figura 6. Degradación progresiva de la muestra con relación de a/c 0.55 
Las probetas de hormigón después de 180 ciclos de exposición a sulfato, presentan daño en la superficie por encima del nivel de solución, esto por los cambios de temperatura y la humedad calibrada de acuerdo a la fases de diagrama de sulfato de sodio, se observa un daño extenso en las probetas expuestas con relaciones de a/c 0.45 (bajos) a comparación con las probetas de hormigón con relación de a/c 0,55 (alto). Se observó también deño considerable en el material impermeabilizante recuplast ladrillo.

Para las muestras impermeabilizadas con recuplat techo se observó recubrimiento crítico de dos pasadas sin mejorar la densidad del material impermeabilizante, el daño consiste en el contacto del agente corrosivo con el hormigón a través del macro poro que no ha sido recubierto completamente, esto inmediatamente por encima del nivel de la solución hasta aproximadamente el nivel de altura de la probeta. El material poliuret para pisos de alto tránsito no sufrió daño, aunque se formaron unas ondulaciones por la radiación solar impuesta en la cámara ambiental.

\section{Pérdida de masa}

Los cambios de masa de las probetas de hormigón expuestas parcialmente a una solución de sulfato de sodio al $6 \%$ se controlaron en los periodos de $0,30,60$, 120, 180 ciclos, para los cilindros impermeabilizados y no impermeabilizados. Para la mayoría de las probetas expuestas a laboratorio, la tasa de pérdida de masa fue baja hasta los 30 ciclos, luego significativamente aceleró hasta el final de la exposición, conforme a la tendencia de la evaluación visual. El cambio de masa más drástico es para las probetas no impermeabilizadas con relaciones de a/c
0.45 , siguiendo con las probetas de relación de a/c 0.55. Las probetas impermeabilizadas con recuplat ladrillo han tenido una tendencia similar de pérdida de masa, mientras los otros materiales restantes han sido constantes.

Los cambios de masa se generaron por encima del nivel de solución, generando desprendimiento en la superficie del hormigón, por cambios de temperatura y evaporación que creó una sobresaturación de la solución de sulfato de sodio. Por lo tanto, los cristales pueden crecer a partir de una solución sobresaturada y ejercer una presión alta a la tracción, lo que conduce al daño y el cambio de masa del hormigón por encima del nivel de la solución. Por lo tanto, el control de la variación de la masa parece ser una indicación útil del desempeño de hormigón expuesto al ataque a sulfatos.

\section{CONCLUSIONES}

El ataque por sulfatos es un tema crítico para la durabilidad de estructuras de hormigón expuestas a medios ricos en sales y entornos cíclicos. La cristalización creada por sales que crecen a partir de una solución sobresaturada es un mecanismo altamente perjudicial. En la sobresaturación hay varios factores que afectan a la cristalización de sales, la transformación de fase de sulfato de sodio de thenardita a mirabilita obedece un diagrama de fases en las variaciones de temperatura y humedad relativa relacionada directamente con entornos climáticos cíclicos y velocidad de evaporación en los materiales de estructura de poros.

El mecanismo de daño de ataque de sulfato de sodio sobre cemento significa un proceso fisicoquímico complejo, incluyendo varias producciones perjudiciales como la formación de etringita y yeso que generan la 
expansión (a través de la reacción química). Asimismo, el crecimiento de los cristales por sobresaturación en los poros por acción capilar. La evaporación y diagramas de fases de sulfato de sodio son un problema crítico que afecta la durabilidad de estructuras de hormigón expuestas a medios ricos en sal y ambientes cíclicos. Las manifestaciones de daño por estos cristales son escalamiento superficial y desprendimiento, que típicamente se acompañan de eflorescencia. Los resultados de pérdidas de masa y las características microestructurales demostraron que el procedimiento de prueba propuesto es factible de replicar. El deterioro se produce debido a la cristalización de la sal con la interacción química potencial entre los iones de sulfato y la pasta de cemento hidratada.

La reducción de relaciones de a/c con un asentamiento de cono de abrams entre 0 $2 \mathrm{~cm}$ no mejoró el rendimiento expuesto a ataque de sulfato de sodio. Una buena práctica para mejorar la durabilidad del hormigón implica una relación de a/c 0.55 que forme hormigones con humedad trabajable y con materiales impermeabilizantes. En los casos de materiales de tratamiento de superficie a base de poliurest para pisos de alto tránsito, alquitrán modificado, recuplast para techo; se consideran adecuados para proteger el hormigón, puesto que su revestimiento es difícilmente penetrado por sulfatos, mitigando así el ascenso capilar en el hormigón; mientras el uso de recuplast para ladrillo no proporcionó una protección adecuada.

El diseño de hormigón que es resistente al ataque de los sulfatos implica la creación de una barrera entre el agua con iones de sulfato y el hormigón con materiales impermeabilizantes revestidos según especificaciones: es útil aplicar materiales impermeabilizantes mejorando su viscosidad en la primera capa de tratamiento; el alquitrán modificado es una alternativa económica y efectiva; aplicar recuplast techo es otra buena opción siempre que los macro poros estén revestidos. El curado del hormigón es esencial para combatir estos ataques y la aplicación de materiales impermeabilizante puede mejorar significativamente la durabilidad del hormigón.

\section{REFERENCIAS}

Administradora Boliviana de Carreteras. (2007). ABC. Manual de carreteras. Bolivia

American Concrete Institute. (2001). ACI 210.2R-01 Guía para la Durabilidad del Hormigón

Araújo, D. D. (2013). Sulfate Attack on Cementitious

Collepardi, by M. (2002). <Ettringite Formation and Sulfate Attack on Concrete.pdf>. 17.

Corral, J. T. (2009). La resistencia a compresión del hormigón, condición necesaria pero no suficiente para el logro de la durabilidad de las obras. Ciencia y sociedad, 4(4), 463-504

Hayner, H. y Bassuoni, M. T. (2012). Ataque Fisico de Sales Sobre el concreto (traducido)

Instituto Boliviano de Normalización y Calidad. (1987). CBH-87, Norma Boliviana, Hormigón armado. Bolivia

Jara Del Rosario, J. W. (2016). Evaluación de la durabilidad del mortero proyectado frente a ataques por sulfato (Master's thesis, Universitat Politècnica de Catalunya)

Ministerio de Fomento, (2011). EHE-08, Introducción de Hormigon Estructural. Con comentarios de los miembros de la Comisión Permanetne del Hormigón. España 
Montoya, P.J. (2010). Hormigón armado: Editorial Gustavo Gili, S.L

Neville, A. (2004). The confused world of sulfate attack on concrete. Cement and Concrete Research, 34(8), 1275-1296. doi: 10.1016/j.cemconres.2004.04.004
UNE-EN-12390-3. (2003). Determianción de la resistencia a compresión de probetas

UNE-EN-12504-2. (2002). Ensayos no destructivos Determinación del índice de rebote 8 